Monatsschrift für Geburtshülfe u. Gynäkologie 1912;35:125-134

\title{
Franz von Winckel †
}

Am 31. Dezember vergangenen Jahres in der Frühe urn 4x/2 Uhr verschied ini Kreise seiner Familie Franz v. Winckel.

Lange Zeit zurückreichender Diabetes mellitus hatte in den letzten Jahren seine frülier so ungebrochene Kraft geschwächt und ein apoplektischer Anfall vor einigen Monaten seine geistigen und körperlichen Kräfte erliebliclx reduziert. Der Tod kam ihm als Erlöser.

Franz v. Winckel wurde geboren am õ. Juni 1837 zu Berleburg in Westfalen als Sohn des Fürstlich Sayn-Wittgensteinschen Hof-und Leibarztes ünd Königl. Preußischen Kreisarztes Ludwig Winckel. Er stammte aus einer Ärztefamilie, auch sein Groß-vater war Arzt. Sein Vater hat sich in der wissenschaftlichen Welt durch seine für die damalige Zeit vorzüglichen Resultate beim Kaisersclmitt einen guten Namen gemacht. Früh schon lernte Winckel die Freuden und Leiden des ärztlichen Berufes aus eigener Anschauung kennen; er erzählte später, als er in Amt und Würden war, gerne und mit Humor von den Praxisfahrten, die er gemeinschaftlich mit seinem Vater in seiner Jugend unter-nommen hat.

Xach Vollendung seiner Studien trat Winckel bei Eduard Martin in Berlin als Assistent ein. Dort verblieb er, bis der erst 27 jährige, der eben erst vor der Habilitation stand, eine Berufung nach Rostock als Ordinarius für Geburtshülfe und Pharmakologie erhielt (1865). Das Lehrfach der Pharmakologie versah Winckel während seines ganzen Rostocker Aufenthaltes. Diesem Umstande verdankt er seine umfassenden Kenntnisse in der Medizinal- und Drogenlehre, die sich allezeit deutlich in seiner Rezeptur ausdrückte. Ungern schied Winckel aus Rostock, das er sehr lieb gewonnen hatte, um 1872 die Leitung der Entbindungs-anstalt in Dresden zu übernehmen. Diese $\Lambda$ vandelte er in eine moderne Frauenklinik mit besonderer gynäkologischer Abteilung um, richtete eine geburtshülfliche Poliklinik und, was damals ganz neu war, in der Klinik ein Internat für Arzte ein, das ihnen die Möglichkeit bot, alle in der Klinik vorkommenden

126

Franz von Winckel $\uparrow$.

geburtshülflichen Ereignisse zu beobachten und unter Anleitung selbständig Operationen auszuführen. Es war das eine vorzügliche Vorschule für die Praxis. Von alien Seiten strömten lernbegierige junge Ärzte herbei. Darunter befanden sich viele Ausländer. namentlich Anierikaner, und so kam es, daß der Name Winckel bald auch im Auslande einen guten Klang hatte. Besonders große Verdienste hat sich v. Winckel in Dresden um die Neuregelung und Organisation des sächsischen Hebammenwesens erworben, welches heute noch als mustergültig organisiert angesehen werden kann.

Seine Berufung nach München erfolgte 1883 als Nachfolger von Heckers. Auch hier war seine erste Tat, die vorher stadtische und Kreisentbindungsanstalt in eine moderne staatliche Frauenklinik umzuwandeln. Mit ihm zog der erste Fachmann für operative Gynäkologie in München em. In seiner neuen Wirkungsstätte gründete er die gynäkologische Gesellschaft und sammelte so Gleichstrebende um sich. Seiner Initiative ist es in erster Linie zuzuschreiben, daß die lang ersehnte deutsche Gesellschaft für Gynäkologie schließlich zustande kam. Er hatte die Freude, den ersten deutschen Gynäkologenkongreß in München 1886 er-öffnen und leiten zu können. 
Am 5. Juni 1907 wurde von seinen Schülern und Freunden in einem feierlichen Akt in der Frauen-klinik sein 70. Geburtstag festlich begangen. Am 1. Oktober desselben Jahres trat er vom Lehrfach zurück. In den letzten Jahren seiner Amtstätigkeit hatten bereits seine sonst unerschöpfliche Arbeitskraft und sein geistiger Schwung durch die schleichende Krankheit gelitten; sie war es auch, die im Verein mit einer Arteriosklerose ihm die letzten 4 Jahre seines Otium cum dignitate wenigstens zeitweise trübte.

Es ist für einen der jüngeren Generation Angehörigen, auch dann, wenn er ihm als Schüler nahe gestanden hat, nicht ganz leicht, dem Lebenswerk v. Winckels ganz gerecht zu werden; denn die wichtigste schöpferische Arbeit v. Winckels liegt zeitlich weit zurück, und die Probleme und Fragestellungen der damaligen Zeit $\Lambda$ varen naturgemä $ß$ ganz andere als in der neueren Epoche. Schon seine ersten Arbeiten als Assistent an der Martinschen Klinik und der ersten Rostocker Zeit verrieten in ihm den gründ-lichen Forscher. Es gait in der damaligen Zeit zunächst durch Sammlung möghchst vieler und exakter Beobachtungen die Grund-lagen für eine bessere Physiologie der Schwangerschaft, der Geburt, des Wochenbetts und des Neugebornen zu schaffen. Er hat mit seinen ersten Arbeiten über „Die Gewichtszunahme der Neu-

Franz von Winckel †. 127

gebornen”, über ,,Die Gewichtsverhältnisse der Schwangeren”, über ..Die Harn- und Stoffwechseluntersuchungen schwangerer Frauen" zu der Frage äußerst wichtige Beiträge geliefert. Auf seine Veranlassung hin wurden zum ersten Male regelmäßige

Temperaturmessungen an gesunden Wöchnerinnen, die bisher nicht üblich gewesen waren, an der Berliner und Rostocker Klinik durchgeführt. Seine in Dresden und Rostock herausgegebenen zwei Bände .,Berichte und Studien” enthalten die Mitteilung wertvoller klinischer

Beobachtungen und Erfahrungen und bringen ein großes statistisches Material für die Häufigkeit des Vorkommens physiologischer Zustände und pathologischer Ereignisse in der Ge-burtshülfe. Als einer der ersten trat er nüt Wärme für die Semmel-we $3 / 4 / 8$ che Lehre ein und führte die Lehre in den von ihrn geleiteten Kliniken praktisch durch. Er hielt bis zu seinem Scheiden aus der Amtstätigkeit an der ausschließlich ektogenen Infektion fest und machte mit unerbittlicher Konsequenz für das Auftreten eines Infektionsfalles den verantwortlichen Assistenten haftbar. Seine Studien über das Puerperalfieber und über die Wochenbetts-erkrankungenfaßte er in demausgezeichnetenWerke ,,DiePathologie und Therapie des Wochenbettes” zusammen. Für die hohe Wert-schätzung des Buches spricht der Umstand, daß es innerhalb kurzer Zeit 3 Auflagen erlebte (1. Auflage Berlin 1866) und ins Holländische und ins Englische übersetzt wurde. Ein großer Wurf war seine ,,Pathologische Anatomie der weiblichen Ge-schlechtsorgane”. Dieses Atlaswerk trug viel zur besseren und allgemeineren Kenntnis der damals noch wenig bekannten Pathologie der weiblichen Geschlechtsorgane bei; es zeichnete sich durch seine vorzüglichen photographischen Reproduktionen in der vorteilhaftesten Weise vor den Abbildungen der damaligen Zeit aus. In die Münchener Zeit fällt die Herausgabe seiner Lehrbücher der Geburtshülfe und der Frauenkrankheiten. Es sind gründliche, mit großer Erfahrung und eminenter Literatur-kenntnis verfaßte Werke und Fundgruben für jeden, der sich genauer mit der Materie beschäftigen will. Sie leiden indes als Lehrbücher darunter, daß sie zu sehr in manche Details eingehen und daß sie für den Studierenden etwas hoch und schwer geschrieben sind. Sein Lehrbuch der Geburtshülfe wurde ins Englische übersetzt und fand speziell in Amerika großen Beifall. In der von Biïlroih und Lücke herausgegebenen „Deutschen Chirurgie” hat Winckel die Erkrankungen der Harnröhre und der Blase übernommen und sie in mustergültiger Weise nach dem damaligen Stand des Wissens bearbeitet (1885). Auch in 9* 
128

Franz von. Winckel $\uparrow$.

dem bekannten ,Handbuche der Therapie” von Penzoldt und Stinzing hat Winckel in der ersten Auflage mitgearbeitet. Er war Herausgeber der gynäkologischen Abteilung der Volkmann-schen Sammlung klinischer Vorträge und hat selbst wertvolle Beiträge in ihnen veröffentlicht. Er hielt große Stücke auf diese Zeitschrift und behielt deren Redaktion auch nach seinem Zurück-tritte voin Amt noch bei. Auch unter deni Herausgeber-kollegium der Münchener medizinischen Wochenschrift befand sich Winckel.

Außerordentlich groß ist die Zahl der kleineren Arbeiten; es seien hier nur einige wenige erwähnt.

1879 beschrieb Winckel zum ersten Male die akute Hämo-globinämie der Neugebornen, die später als Winckehche Krankheit in der Literatur bekannt wurde. Eine andere von ihr $\cap$ zum ersten Male beschriebene Anomalie ist die Kolpohyperplasia cystica gravidarum.

Bedeutungsvoll für die Frage der Selbstinfektion ist die Arbeit Winckels ,,Die Bedeutung präzipitierter $\mathrm{G}^{1} / 8$ burten” München 1884 . In seinen Untersuchungen , Über die Dauer der Schwangerschaft" ging Winckel von einem bisher noch nicht be-nützten Prinzipe aus, ungewöhnlich stark entwickelte Kinder zur Bestimmung der Schwangerschaftsdauer zu verwenden, und ge-langte zu wichtigen Resultaten, die durch viele spätere Nachunter-suchungen bestätigt wurden. In den letzten Jahren seiner Aktivität wendete sich Winckel mit besonderer Vorliebe Forschungen über die $\mathrm{G}^{1} / 8$ nese menschlicher Mißbildungen $\mathrm{zu}$; die anatomische Sammlung der Klinik war angefüllt mit Mißbildungen aller Art, die ihm von weither von ehemaligen Schülern und praktischen Ärzten zugeschickt wurden. In zwei Volkmannscheii Vorträgen ,,Über Einteilung und Entstehung der Mißbildungen” berichtet er über die Früchte seiner Studien. Er glaubte die Mehrzahl der Mißbildungen auf amniotische Fäden zurückführen zu können.

Diese Auslese bereits gibt einen Einblick in die schrift-stellerische Fruchtbarkeit Winckels; es sind Proben seines tief gründlichen Wissens und seiner ernsten Forschertätigkeit, aber keines ist für Winckel so charakteristisch, wie die erst nach seinem Zurücktritte vom Amt 1909 erschienene ,,AllgemeineGynäkologie”. Kein anderer wohl, wie er, vereinigte so viel allgemeines Wissen in sich, um zum ersten Male an dieses schwierige und wichtige Problem heranzugehen. Das Buch ist entstanden aus einer Reihe von Vorlesungen, die Winckel in den Jahren 1884-1887 und in den 90 er Jahren hielt. Die Arbeit hätte sicherlich noch mehr BeFranz von Winckel $\uparrow$.

129

wunderung hervorgerufen, wenn sie in der Zeit, in der sie ent-standen war, veröffentlicht worden ware; sie erschien 15 Jahre zu spat. Damals als die Vorlesungen gehalten wurden, war z. B. die Frauenbewegung, für die Winckel von jeher mit der größten Wärme eingetreten ist, noch im Beginn; heute sind die Forderungen, die damals vielfach als Auswüchse unweiblichen Empfindens und als Utopien verlacht wurden, zum großen Teile längst erfüllt. Es leuchtet aus dem Buche ein so tiefes Verständnis für die weibliche Psyche und ein so edles Mitgefühl für die Leiden der Frauen hervor, es spricht ein weiter und klarer Blick und eine so große Erfahrung, daß es eine Wonne ist, in ihm zu lesen. Nirgends tritt sein um-fassendes Wissen und seine eminente Belesenheit so deutlich zutage wie dort. Ein weiteres Charakteristikum Winckels ist, daß er sich mit besonderer Vorliebe auch mit den einem Fachmann etwas ferner liegenden Gebieten beschäftigt. Jedes Semester wurde mit einem Vortrag allgemeinen Inhaltes feierlich eröffnet; es waren die Früchte literarischer Studien während der Ferien. Bald war es eine 
eingehende Besprechung und Würdigung eines wichtigen Buches, z. B. For els sexuelle Moral und Hygiene, bald machte er an der Hand eines bedeutsamen Reisewerkes seine Hörer mit den Sitten und medizinischen Gebräuchen und Anschauungen wilder Volksstämme bekannt, dann wieder behandelte er eine allgemeine medizinische Frage. Stets riefen seine allgemeinen Vorträge in höchstem Grade das Interesse und den Beifall seiner Hörer hervor. E1/8er dieser einleitenden Vorträge ,,Shakespeares Gynäkologie” ist in Volhmanns Sammlung in extenso erschienen.

Den Abschluß seiner Lebensarbeit bildete sein großes ,Hand* buch der Geburtshülfe”, das eben in dem Augenblicke vollendet dastand, als er von seinem Amte zurücktrat. Das Werk hat manche Schwächen: Verschiedenwertigkeit der Mitarbeiter, zu sehr ins Detail gehende Einteilung, zu beträchtliche Ausdehnung. Trotz dieser Schwächen muß es indes als ein stolzes Werk deutscher Arbeit und Gründlichkeit angesehen werden, wie die Welt-literatur kein zweites aufzuweisen vermag. Winckel selbst hat es als eine Art Krönung seines Lebenswerkes angesehen.

v. Winckel war kein stürmischer Neuerer, der in genialer Intuition Altes niederreißt und Neues, Vollkommeneres an seine Stelle setzt. Er war vielmehr der exakt und peinlich genau arbeitende Forscher, der alles, auch das Kleinste beobachtet, überall sein Material sammelt und nach und nach zu einem festen Baue fügt. Vermöge seines vielbewunderten und untrüglichen Ge130

Franz von Winckel $\uparrow$.

dächtnisses vermochte er alles, was er je gelesen hatte. zu behalten. Er hatte in hoheni Grade die Fähigkeit, in schöner und fließender Rede seinen Gedanken Ausdruck zu verleihen. Sein Wissen hatte etwas Enzyklopädisches und glich in seinem Umfange der Größe seiner Bibliothek, die ehedem sein ganzes Haus vom Kellerraum bis unter das Daeh anfüllte. Was Winckel als Arzt besonders aus-zeichnete, das war, daß er menials als reiner Spezialist kurierte, sondern stets den Zusammenhang der Genitalorgane mit deni ge-samten Organismus im Auge behielt, daß er mit einer peinlich ge-nauen, manchmal ans Pedantische grenzenden Sorgfalt die Stellung der Diagnose und Indikation betrieb. Seine Kranken hatten zu ihm ein unbegrenztes Vertrauen, und da, wo seine Kunst nicht mehr zu heilen vermochte, wirkte er durch seinen suggestiven Einfluß beruhigend auf sie ein. Es lag die Ursache davon wohl in seinem sicheren Auftreten und in seiner in sich abgeschlossenen und ge-festigten Persönlichkeit. Er war seiner selbst so sicher, daß er sich wenig durch äußere Momente beeinflussen ließ, und diese Eigenschaft verlieh ihm im höheren Alter manchmal etwas Un-nahbares. Auf sein Wort konnte man sich felsenfest verlassen, daran gab es kein Rütteln und Deuteln. Sehr ausgeprägt war sein Ge-rechtigkeitssinn, das gait so wohl im objektiven Sinn gegen andere als auch im subjektiven, denn er konnte jemand, von dem er sich unbillig behandelt glaubte, ehrlich hassen. Alle, die ihm näher standen, verehrten seinen hohen Sinn und seine vornehme Ge-sinnung. Die Zeiteinteilung war ein Geheimnis Winckels. Auf den Glockenschlag traf er zur Arbeit ein, jede Minute des Tages war vorher bestimmt und ausgenutzt, und nur besonders vor-dringliche Dinge vermochten ihn von der gemachten Tages-einteilung abzubringen. Nur so ist es zu erklären, daß es ihm mög-lich war, neben der erdrückenden Last der täglichen Arbeit, der Tätigkeit als Lehrer, als Eakultätsmitglied, als Operateur, als viel-gesuchter Frauenarzt und Consiliarius eine derartige wissenschaft-hche Produktivität zu entfalten.

In den Mußestunden beschäftigte er sich gerne mit belle-tristischer Literatur. Es ist kaum ein Werk von Bedeutung er-schienen, das nicht in seiner Bibliothek gewesen ware und das er nicht gelesen hätte; er wußte auch hierin überall Bescheid. In Gesellschaft konnte er anregend und mit 
sprudelndem Humor erzählen; in der Münchener Gesellschaft der ,,Zwanglosen”, deren Vorsitzender er lange Zeit war, legte er häufig Proben seines Sinnes für Witz und Humor ab. Franz von Winckel $\uparrow$.

131

Unübertrefflich war er als Gatte und Vater. Der überaus glücklichen Ehe entsprossen 1 Sohn und 4 Töchter. Wenige Monate noch vor seinem Tode wurde ihm eine Urenkelin geboren. Schwer traf ihn der frühzeitige Tod seines Schwiegersohnes Walther (1907), der in treuer Pflichterfüllung als Arzt sich aufopferte.

Nun ist sein Lebenswerk vollendet; der rastlose Geist ist zur Ruhe gegangen, sein Körper ist durch des Peuers heilige Plamme aufgelöst. Wir standen trauernd an seineni Sarge; denn einer unserer Größten und Besten ist nicht mehr.

L. Seitz, Erlangen.

Vor mehr als 50 Jahren - als ich als Knabe aus der Pension ins Elternhaus kam -• sah ich Tag für Tag meinen Vater beim Verlassen der klinischen Räume von einem jungen Assistenten geleitet, der in eifrigstem Gespräch des Altmeisters Worten lauschte,-- er war auch in unsere Familie wie ein Sohn aúgenommen: das war Franz Winckel! Ein Zufall ist es gewesen, daß ich ihn erst wieder-sah, als er auf der Naturforscher-Versammlung in Hamburg 1876 mit seiner berühmten Rede für die ärztliche Portbildung eintrat. Seitdem hat sich ein reger Verkehr zwischen uns entwickelt. Winckel war wohl unter den deutschen Klinikern am häufigsten mein Gast in der Elsasser Straße. Ich sah ihn in Dresden, dann in München, auf zahlreichen Versammlungen und Kongressen in Deutschland und im Ausland, stets von neuem fasziniert von seiner überreichen wissenschaftlichen Tätigkeit, von seiner be-zaubernden persönlichen Liebenswürdigkeit. So hat er mir mit hundertfältigen Zinsen heimgezahlt, was er seinerzeit von meinem Vater, in meinem elterlichen Haus empfangen!

Mit tiefer Bewegung mußte ich in den großen Münchener Tagen im Juni 1911 wahrnehmen, daß seinem Erdenwallen ein nicht mehr femes Ziel gesetzt war! Er ist von schwer empfundenen Leid erlöst: Have! Have!

Neben den treu empfundenen Worten seines Schülers Ludwig Seitz möchte ich hier nur nach zwei Richtungen hin noch die großen und unvergeßlichen Verdienste Franz von Winckéls besonders hervorheben. Er hat wohl die Bedeutung einer systematischen Portbildung des Arztes auch über die Zeit des akademischen Studiums hinaus - - nicht als erster - - erkannt, denn seit jeher zogen besonders deutsche Ärzte nach Vollendung ihrer offiziellen Ausbildungszeit hinaus in die Zentren großer Hoch-

132

Franz von Winckel $\uparrow$.

schulen, zu hervorragenden Forschern und Lehrern. Aber $\Lambda$ vie wenigen nur erlaubten die Mittel ein längeres Verweilen, eine intensivere Arbeit in der Feme ? Winckel skizzierte zuerst in seinem Hamburger Vortrag 1876 nacli seinen in Rostock und in Dresden gewonnenen Erfahrungen die Plane zu ärztlichen Fortbildungs-kursen, $\Lambda$ velche heute in alien Hochschulen eingerichtet sind und in kurzer Zeitspanne es ermöglichen, daß der strebsame Arzt sich mit den in so rascher Entwicklung begriffenen Methoden und Forschungen auf alien Gebieten der vielgestaltigen medizinischen Wissenschaft bekannt, gegebenen Falles in mehrjährigen Wiederholungen auch vertraut niacht. Mcht einen $\alpha$ oberflächlichen Spezialistentum von zweifelhafter Berechtigung -, nein, der gründ-lichsten Weiterbildung sollte Anregung und Ermöglichung geschafi'en werden! Wie er selbst ein vielseitiges Wissen und gründliche Vertrautheit in intensivster Ausdehnung anstrebte, so verlangte Winckel für die Gesamtheit der Ärzte, besonders 
aber für die Spezialisten, die Förderung allgemeiner Kenntnisse und rast-lose Teilnalime an dem Fortschreiten der ÎSГ aturwissenschaften und der Medizin!

Ein anderes Verdienst des Heimgegangenen war die Anregung zur Förderung der persönlichen Beziehungen und die persön-liche Kenntnisnahme zunächst ärztlicher, dann aber allgemein kultureller Verhältnisse und Einrichtungen über die Grenzen nicht nur der speziellen Heimat, über das ganze Erdenrund! Die Urbanität seiner liebenswürdigen Persönlichkeit, seine polyglotte Bildung, seine unermüdliche Beweglichkeit kannten keine Mühe, scheute keine Opfer an persönlicliem Behagen, wenn es gait, die in wissenschaftlichem Verkehr angeknüpften Beziehungen jenseiti $3 / 8$ der schwarzweißroten Grenzpfähle, über feme Meere weiter zu spinnen und durch sie gestützt alte und neue Kulturländer zu durchforschen, nicht nur als Arzt, als Kulturforscher zu durch-dringen. Welche großartigen, von scharfer Beobachtung und köstlichem Humor getragenen Schilderungen brachte er davon nach Haus, zur Belehrung und Unterhaltung am häuslichen Herd, in den weiten Kreisen seiner Kollegen und Freunde! Immer wieder war ich erfreut, wenn ich an der Moskwa, im Kaükasus, im Norden und Süden unseres Kontinents, in den Vereinigten Staaten von Nordamerika Winckels Spuren fand. Überall hatte er warme Freundschaften hinterlassen, ein verehrungsvoiles Andenken! Und nicht nur die Manner, ganz besonders die Frauen wurden nicht müde, seinen liebenswürdigen Umgang, seine belehrende und dabei bezaubernd anregende Unterhaltung zu rühmen und zu preisen!

Franz von Winokel $\uparrow$.

133

Als ein anderes Mittel zu demselben Zweck anerkannte Winckel den persönlichen Austausch von Eríahrungen, Studien und Anschauungen auf wissenschaftlichen Versammlungen und $3 / 4$ longressen. Unermüdlich von den frühesten Morgenstunden bis zum Abend verfolgte er die Verbandlungen, jedesmal brachte er selbst einen bedeutungsvollen Beitrag, er war einer der gewandtesten und schlagfertigsten in der Diskussion. Unter vollster Würdigung des Lehrwertes der eigenen Anschauung hat Winckel als einer der ersten ganze Sammlungen wertvoller Präparate weithin mit sich geführt. Die Originalien seines Atlas haben wir in ihrer allmählichen Einrichtung auf verschiedenen Versaimnlungen und an ihnen die mustergültige Aufmachung kennen gelernt. Und mit wie viel uner-müdlicher Beweitwilligkeit ging er in ihre Erörterung ein. Wie erstaunlich frisch war er dann am Abend, wenn er mit seinem sprühendem Humor die ihm andächtig lauschende Schar der alten und jungen mit den scharf beobachteten Erlebnissen als Lehrer und Porscher, als Kunstkenner und vielerfahrener Gesellschafter, als glänzender Erzähler unterhielt und bis zum anbrechenden Morgen um sich fesselte!

Franz von Winckels grundlegende Arbeiten bleiben als Eck-steine in dem Unterbau der Geburtshülfe und. Gynäkologie bestehen. Möge auch das Andenken an seine liebenswürdige Persönlichkeit, an den trefflichen Mann vorbildlich auf kommende Generationen übergehen! Der Kreis derjenigen, welche ihn in den Dezennien seiner Vollkraft kannten und ihm nahestanden, lichtet sich natur-gemäß; wir trauern ihm nach, uns wird er in der Erinnerung lebendig vor der Seele stehen als leuchtendes Beispiel eines liebens-werten, vornehmen Mannes und treuen Ereundes!

A. Martin.

Hauptarbeiten von F. von Winckel.

Studien über den Stoffwechsel bei Schwangeren. I860. Die Pathologie und Therapie des Wochenbettes. 1866. (3. Aufl. 1868.) Klinische Beobachtungen zur Pathologi $\theta$ der Geburt. 1869. Bericht $\theta$ und Studien aus der Kgl. Entbindungsanstalt in Dresden. (3 Bde.) Die Krankheiten der weiblichen Harnröhre und Blase. 1877, in der Chrrurgi $\theta$ von Billroth und Lücke. 
Aus 1878-81 stammen:

Die Pathologi $\theta$ der weiblichen Sexualorgane, in Lichtdruckabbildungen nach der Natur in Originalgröße.

134

Franz von Winckel $\uparrow$.

Üb $\beta r$ die B $\theta d e u t u n g$ präzipierter Geburten für die Ätiologie des Puerperal-fiebers, erne

Festschrift zum 50 jährigen Doktorjubiläum von Seitz.

1886 erschien das Lehrbuch der Frauenkrankheiten (2. Aufl. 1893, eine englische Übersetzung 1890).

Lehrbuch der Geburtshülfe. 2. Aufl. 1893.

Behandlung der von den weiblichen Genitalien ausgehenden Entzündungen des Bauchfells. $1 \S 97$ im Handbuch der Pathologie von Penzoldt und Stintzing.

Seit 1883 war W. Mitarbeiter an der Allgemeinen deutschen Biographie vom Buchstaben M an, außerdem gab er im Verein mit v. Bergmann und W. Erb, jetzt Friedrich Mutter - München, die neue Folge von klinischen Vorträgen von Volkmann heraus.

Handbuch der Geburtshülfe im. Verlage voń J. J. Bergmann in Wiesbaden. 1909.

Die kgl. XJniversitätsfrauenklinik zu München. 1884-90.

Im übrigen verweisen wir auf die ausführliche Angabe der Winckelschen Arbeiten im dritten Band der Geschichte der Geburtshülfe von Siebolä, bearbeitet von Dohrn. 